\title{
Composición y abundancia de artrópodos epígeos del Parque Nacional Llanos de Challe: impactos del ENOS de 1997 y efectos del hábitat pedológico
}

\author{
Abundance and composition of epigean arthropods from Llanos de Challe National Park: \\ impacts of ENSO-1997 and effects of the pedological habitat
}

JORGE CEPEDA-PIZARRO*, JAIME PIZARRO-ARAYA \& HERNÁN VÁSQUEZ

Laboratorio de Entomología Ecológica, Departamento de Biología, Universidad de La Serena, La Serena, Chile; *e-mail para correspondencia: jcepeda@userena.cl

\begin{abstract}
RESUMEN
Mediante el uso de trampas de intercepción, se estudió el efecto de la precipitación y el tipo de hábitat sobre la denso-actividad del ensamble de artrópodos epígeos -en particular Tenebrionidae- del Parque Nacional Llanos de Challe. Este parque está ubicado en el desierto costero transicional de Chile, en la III Región de Atacama. Los muestreos se realizaron durante tres días por mes (septiembre, octubre y diciembre) en el período de máxima actividad biológica del sistema y en los años 1989 (año no-ENOS seco), 1997 (año ENOS intenso) y 2000 (año no-ENOS húmedo). En las comparaciones se utilizaron dos sitios pedológicamente contrastantes: un hábitat dunario costero y un hábitat pedregoso interior. Se registró la presencia de 15 órdenes de Arthropoda. Los hexápodos constituyeron > $95 \%$ del total de los especímenes capturados (9.065 individuos), siendo Collembola $(36,1 \%)$ y Coleoptera $(29,8 \%)$ los órdenes con mayor representatividad numérica. El número de órdenes con representantes activos varió ligeramente entre años: 13 órdenes se registraron en 1989 (44\% de precipitación bajo el promedio), 15 en 1997 (443\% sobre el promedio) y 11 en 2000 (52\% sobre el promedio). El efecto del evento ENOS se reflejó claramente en la contribución numérica en la mayoría de los taxa registrados. Aunque más sutilmente, este efecto también se reflejó en la composición del ensamble de taxa dominantes y entre hábitat pedológicos, particularmente con Tenebrionidae y Formicidae. Especialmente en las dunas costeras, Tenebrionidae dominó claramente el ensamble de artrópodos epígeos, siendo Gyriosomus Guérin-Méneville el género más diverso y abundante. La dominancia de Gyriosomus plantea un conjunto de interrogantes respecto de su nivel de endemismo, diversidad y distribución de sus especies, y el papel funcional de estas en el ecosistema estudiado.
\end{abstract}

Palabras clave: zonas áridas, desiertos costeros, artrópodos epígeos, Tenebrionidae, ENOS.

\begin{abstract}
By using pitfall traps, the effects of rainfall and habitat type on the denso-activity of epigean arthropods Tenebrionidae in particular- inhabiting at Llanos de Challe National Park were investigated. This park is located in the Chile's transitional coastal desert, Atacama's III Region. Sampling was conducted during three days per month (september, october and december) in the period of maximal biological activity of the system, and in 1989 (dry no-ENSO year), 1997 (intense ENSO-year), and 2000 (damp no-ENSO year). Two pedological contrasting sites were used to compare the effects of the factors being examined: a coastal dune habitat and an interior stony habitat. The presence of 15 orders of Arthropoda was recorded. Hexapods made the >95\% of total specimens captured (9,065 individuals), with Collembola $(36.1 \%)$ and Coleoptera $(29.8 \%)$ being the orders with the highest numerical representativeness. The numbers of orders with active members varied slightly among years: 13 orders were recorded in 1989 (44\% rainfall below the average), 15 in 1997 (443\% rainfall above the average), and 11 in 2000 (52\% above average). The effect of the ENSO event was clearly reflected in the numerical contribution both for the years studied and for most of the recorded taxa. Although more subtlety, this effect was also reflected in the assemblage composition of dominant taxa and between pedological habitats, particularly in regard to Tenebrionidae and Formicidae. Specially in the coastal dunes, Tenebrionidae clearly dominated the assemblage of epigean arthropods, being Gyriosomus Guérin-Méneville the most abundant and diverse genus. The dominance of Gyriosomus sets a number of questions regarding the levels of endemism, species diversity and distribution, and their functional role in the ecosystem studied.
\end{abstract}

Key words: arid zones, coastal deserts, epigean arthropods, Tenebrionidae, ENSO. 


\section{INTRODUCCIÓN}

Por su posición geográfica y representatividad, el Parque Nacional Llanos de Challe ha sido considerado un sitio relevante en términos de la conservación de la diversidad biológica del país (Muñoz et al. 1996, Moreno et al. 2002). Este parque fue incorporado al Sistema Nacional de Áreas Silvestres Protegidas del Estado (SNASPE) de Chile en 1994 con el fin de proteger y conservar unidades naturales representativas del desierto costero transicional, franja que constituye el límite septentrional del foco de diversidad vegetal ("hotspots") reconocido para Chile central (Cowling et al. 1996, Gaston 2000). Específicamente, el Parque Nacional Llanos de Challe protege y conserva la biota del desierto costero de Huasco (según Gajardo 1993).

La precipitación anual promedio que recibe el parque es baja y muy variable; sin embargo, existen años donde el valor promedio es ampliamente superado. Estos aumentos pluviométricos generalmente están relacionados a incursiones en el territorio chileno del evento ENOS (El Niño-Oscilación del Sur) (Romero \& Garrido 1985, Jaksic 1998). En los últimos años, una probable conexión del evento ENOS con fenómenos asociados al cambio climático y sus impactos sobre los ecosistemas terrestres costeros, han llamado la atención de diferentes investigadores, particularmente de aquellos interesados en las respuestas de la flora y los micromamíferos (Meserve et al. 1995, 2003, Jaksic 1998, 2001, Lima et al. 1999, Gutiérrez et al. 2000a, 2000b, Holmgreen et al. 2001, Yunger et al. 2002, Gutiérrez \& Meserve 2003). A la fecha, la representatividad en los estudios de otros grupos biológicos -como los artrópodos- es casi inexistente (Cepeda-Pizarro et al. en prensa).

Se ha documentado que, bajo condiciones favorables de precipitación como las que ocurren bajo el evento ENOS, los artrópodos que habitan los ambientes áridos pueden alcanzar densidades poblacionales elevadas (Ahearn 1971, Thomas 1979, Sheldon \& Rogers 1984, Parmenter et al. 1989a, 1989b, Cigliano et al. 1995, Polis et al. 1997, Carpaneto \& Fattorini 2001, 2003, Deslippe et al. 2001, Giraldo \& Arellano 2003, Giraldo et al. 2004).
La ocurrencia del ENOS en 1997 (año ENOS intenso, Santibáñez \& Uribe 1999, Muñoz-Schick et al. 2001) y antecedentes reunidos en los años previos (Cepeda-Pizarro et al. en prensa) proporcionaron la oportunidad para evaluar su efecto sobre el ensamble de artrópodos epígeos de un sector representativo y protegido del desierto costero transicional de Chile. Los objetivos del trabajo fueron: (1) documentar, a nivel ordinal, la composición taxonómica de Arthropoda y las variaciones de su abundancia relativa en las fases árida y húmeda del sistema; (2) documentar el efecto del evento ENOS del año 1997 sobre la densoactividad de Arthropoda, y en particular de Tenebrionidae; y (3) evaluar, bajo el evento ENOS, el efecto del hábitat pedológico sobre las variaciones de la denso-actividad de coleópteros epígeos y de tenebriónidos en particular.

\section{MATERIALES Y MÉTODOS}

\section{Sitio de estudio}

El Parque Nacional Llanos de Challe se encuentra ubicado en el desierto costero de Huasco $\left(28^{\circ} 13^{\prime} \mathrm{S}, 71^{\circ} 04^{\prime} \mathrm{O}\right)$. Se extiende por los llanos y serranías costeras e interiores de la Provincia de Huasco, en la Región de Atacama (Chile). Tiene una extensión de $\sim 46,000$ ha e incluye el área de Llanos de Challe y parte de la bahía de Carrizal Bajo. Los cerros más importantes son El Buitre (1.386 m de altitud), Los Chinches (1.180 m de altitud), El Molle (904 m de altitud) y El Toro (575 m de altitud). Se encuentra inserto en la cuenca AlgarrobalCarrizal, la que carece de cursos permanentes de agua superficial. Sin embargo y dadas las características del sustrato, lluvias esporádicas e intensas pueden originar flujos superficiales efímeros que bajan por las quebradas, en algunos casos torrencialmente. El suelo es arenoso en la costa representado por dunas estabilizadas y móviles en la franja costera, franco-arenoso en el llano y franco-ripioso en las quebradas. La vegetación está representada por las asociaciones Heliotropium stenophyllum-Oxalis gigantea, Encelia tomentosa-Nolana paradoxa y por Sarcocornia fructicosa-Juncus acutus en los sectores de suelo salobre (Gajardo 1993). 
Para la realización de este trabajo se seleccionaron dos ambientes pedológicos contrastantes, identificados como: hábitat dunario costero (HDC) y hábitat pedregoso interior (HPI). Ambos se encuentran separados por cerros bajos (690-775 m de altitud) orientados paralelamente a la línea de playa. El HDC forma parte de la franja costera del Parque, tiene unos 0,8-1,2 km de ancho, presenta suelos arenosos estables, con una ligera pendiente ascendente hacia el lomaje que bordea su flanco oriental y que lo separa del llano interior del Parque. El HPI se encuentra al interior del Parque, separado del HDC por los cerros ya mencionados. Los sitios de monitoreo del HPI se instalaron en la Quebrada de Pedernales debido a la presencia de una cubierta vegetal bien establecida. La quebrada es ancha, de pendiente suave y poco profunda, con una orientación N-S. El suelo es aluvial, de textura arenoso-pedregosa. La flora presente en el HDC y HPI está indicada en la Tabla 1. La precipitación anual promedio que recibe el área es $\sim 40 \mathrm{~mm}$ (Toledo \& Zapater 1989), con una elevada variabilidad entre años y una extensa fase árida (Tabla 2). En los años de este estudio el registro de la precipitación fue $44 \%$ bajo el promedio (1989, año noENOS seco, 22,5 mm), $443 \%$ sobre el promedio (1997, año ENOS intenso, 219,5 $\mathrm{mm}), 52 \%$ sobre el promedio (2000, año noENOS húmedo, 61,5 mm) (Tabla 2).

\section{Metodología y diseño del muestreo de Arthro- poda}

Se utilizaron 20 trampas de intercepción dispuestas en parcelas de 4 x $5 \mathrm{~m}$ según el diseño de trampa y forma de emplazamiento en el terreno descritos en Cepeda-Pizarro et al. (en prensa). Siguiendo a Armesto et al. (1993) y Underwood (1999), se instalaron tres parcelas (réplicas) por cada tipo de hábitat (HDC versus HPI). Las parcelas se ubicaron separadas una de otra por 1,2-1,5 km. Para el caso del HDC, la posición de las parcelas fue paralela a la línea de la costa; para el caso del HPI, las parcelas se ordenaron en sentido N-S en la Quebrada Pedernales. Las trampas operaron durante tres días en los meses de septiembre, octubre y diciembre de 1989 (año no-ENOS seco), 1997 (año ENOS intenso) y 2000 (año no-ENOS húmedo). El material capturado fue retirado, limpiado y conservado en alcohol glicerinado $(70 \%)$ hasta el momento de su procesamiento; el material recolectado está depositado en la colección del Laboratorio de Entomología Ecológica de la Universidad de La Serena (LEULS). Para la nomenclatura de los taxa a nivel ordinal se siguió a Artigas (1994) y a Kulzer (1954, 1959), Peña (1966, 1980) y Flores (1997) para el nivel genérico y específico de Tenebrionidae.

La relación entre precipitación y distribución de la abundancia relativa porcentual de Arthropoda fue analizada en el HDC, de acuerdo a antecedentes reportados en Cepeda-Pizarro et al. (en prensa). La relación entre tipo de hábitat y distribución de la abundancia fue, a su vez, examinada durante los años húmedos (e.g., ENOS intenso y noENOS húmedo), de acuerdo a la mayor abundancia de individuos registrados en esos años respecto del año seco. La abundancia del año seco fue muy baja en ambos hábitats, razón por la cual no fue considerado en el análisis. Los resultados de la medición del año ENOS intenso fueron evaluados a través de un ANDEVA para mediciones repetidas (Underwood 1999). En el análisis se usó el programa Statistix, versión 2 (Anónimo 1996). Previo al análisis, los datos fueron logarítmicamente transformados (ln).

\section{RESULTADOS}

\section{Distribución de las abundancias relativas de} Arthropoda y efecto de la precipitación

Considerando los 3 años muestreados, en el sector costero del Parque (HDC) se registró la presencia de 15 órdenes de Arthropoda, con seis de ellos presentando dominancias $\geq 3 \%$, siendo Collembola $(36,1 \%)$ y Coleoptera $(29,8 \%)$ los órdenes con mayor representatividad numérica (Tabla 3). La representatividad de Coleoptera aumenta al $\sim 47 \%$ si Collembola es eliminado del análisis. Les siguieron en dominancia, Diptera $(9,9 \%)$, Lepidoptera (adultos y larvas) $(7,5 \%)$, Hymenoptera (5,7 \%) y Acarina Parasitengonae $(3,0 \%)$. El número de órdenes con representantes activos varió ligeramente entre años: 13 órdenes se registraron en el año no-ENOS seco, 15 en el año ENOS intenso y 11 en el año no-ENOS húmedo. Sin embargo, el 
TABLA 1

Especies vegetales presentes en el Parque Nacional Llanos de Challe (III Región, Chile)

Plant species present in the Llanos de Challe Nacional Park (Region III, Chile)

\begin{tabular}{|c|c|c|c|}
\hline \multirow[t]{2}{*}{ Hábitat } & \multicolumn{3}{|c|}{ Taxa } \\
\hline & Familia & Especie & Forma de vida* \\
\hline \multirow[t]{17}{*}{ HDC } & Alliaceae & Leucocoryne spp. & $\mathrm{H}$ \\
\hline & Amaryllidaceae & Rhodophiala bagnoldii (Herb.) Traub & $\mathrm{H}$ \\
\hline & & Rhodophiala phycelloides (Herb.) Hunz & $\mathrm{H}$ \\
\hline & Apocynaceae & Skytanthus acutus Meyen & $\mathrm{F}$ \\
\hline & Bignoniaceae & Argylia radiata (L.) D. Don. & $\mathrm{H}$ \\
\hline & Boraginaceae & Heliotropium spp. & $\mathrm{H}$ \\
\hline & Chenopodiaceae & Atriplex sp. & $\mathrm{H}$ \\
\hline & Compositae & Bahia ambrosioides Lag. & $\mathrm{F}$ \\
\hline & & Encelia canescens Lam. & SF \\
\hline & Cruciferae & Schizopetalon maritimum Barnéoud & A \\
\hline & Hyacinthaceae & Oziroe biflora (Ruiz et Pav.) Rabean & $\mathrm{H}$ \\
\hline & Ledocarpaceae & Balbisia peduncularis (Lindl.) D. Don. & $\mathrm{F}$ \\
\hline & Malvaceae & Cristaria molinae Gay & $\mathrm{H}$ \\
\hline & Nolanaceae & Nolana spp. & $\mathrm{H}$ \\
\hline & Onagraceae & Oenothera coquimbensis Gay & A \\
\hline & Oxalidaceae & Oxalis gigantea Barnéoud & $\mathrm{H}$ \\
\hline & Portulacaceae & Cistanthe longiscapa Carolin ex Hershk & $\mathrm{H}$ \\
\hline \multirow[t]{32}{*}{ HPI } & Alliaceae & Leucocoryne spp. & $\mathrm{H}$ \\
\hline & Alstroemeriaceae & Alstroemeria spp. & $\mathrm{H}$ \\
\hline & Amaryllidaceae & Rhodophiala bagnoldii (Herb.) Traub & $\mathrm{H}$ \\
\hline & Bignoniaceae & Argylia radiata $($ L.) D.Don & $\mathrm{H}$ \\
\hline & Boraginaceae & Cordia decandra Hook. et Arn & FT \\
\hline & & Heliotropium linariifolium Philippi & $\mathrm{H}$ \\
\hline & & Heliotropium sp. & $\mathrm{H}$ \\
\hline & Caesalpiniaceae & Balsamocarpon brevifolium $\mathrm{Clos}$ & $\mathrm{F}$ \\
\hline & & Caesalpinia angulata (Hook. et Arn.) Baill. & $\mathrm{F}$ \\
\hline & Chenopodiaceae & Atriplex sp. & $\mathrm{H}$ \\
\hline & Compositae & Chaetanthera glabrata (DC.) F.Meigen & A \\
\hline & & Encelia canescens Lam. & SF \\
\hline & & Helenium atacamense Cabr. & A \\
\hline & & Perityle emoryi Torr & A \\
\hline & & Senecio sp. & $\mathrm{H}$ \\
\hline & Frankeniaceae & Frankenia chilensis $\mathrm{K}$. Presl. & $\mathrm{S}$ \\
\hline & Ledocarpaceae & Balbisia peduncularis (Lindl.) D. Don & $\mathrm{F}$ \\
\hline & Malvaceae & Cristaria glaucophylla Cav. & $\mathrm{H}$ \\
\hline & Nolanaceae & Nolana spp. & $\mathrm{H}$ \\
\hline & Onagraceae & Oenothera coquimbensis Gay & A \\
\hline & Oxalidaceae & Oxalis gigantea Barnéoud & $\mathrm{F}$ \\
\hline & Papaveraceae & Argemone hunnemannii Otto et A.Dietr & A \\
\hline & Papilionaceae & Adesmia spp. & $\mathrm{H}$ \\
\hline & Portulacaceae & Cistanthe cachinalensis Philippi & A \\
\hline & & Cistanthe longiscapa Carolin ex Hershk & A \\
\hline & Rubiaceae & Cruckshanksia hymenodon Hook. et Arn & $\mathrm{H}$ \\
\hline & & Cruckshanksia pumila Clos & A \\
\hline & Solanaceae & Lycium sp. & $\mathrm{F}$ \\
\hline & & Phrodus microphylus (Miers) Miers & $\mathrm{F}$ \\
\hline & & Schizanthus candidus Lindl & A \\
\hline & Tecophilaeaceae & Zephira elegans D. Don & $\mathrm{H}$ \\
\hline & Violaceae & Viola pusilla Poepp & A \\
\hline
\end{tabular}

Clave de abreviaciones: $\mathrm{HDC}=$ hábitat dunario costero; $\mathrm{HPI}=$ hábitat pedregoso interior*; $\mathrm{F}=$ arbusto, $\mathrm{S}=$ sufrútice, $\mathrm{H}=$ hierba perenne, $\mathrm{B}=$ hierba bianual, $\mathrm{A}=$ hierba anual (clasificación tomada de Marticorena et al. 2001)

Key to abbreviations: $\mathrm{HDC}=$ coastal dune habitat, $\mathrm{HPI}=$ inland stony-coarse habitat*; $\mathrm{F}=$ shrub, $\mathrm{S}=$ sufrutice, $\mathrm{H}=$ perennial herb, $\mathrm{B}=$ biannual herb, $\mathrm{A}=$ annual herb (classification taken from of Marticorena et al. 2001) 
TABLA 2

Registro pluviométrico en la cuenca de Algarrobal-Carrizal Bajo durante1987-2000 (Estación Hidrológica Canto del Agua, 289' S, $70^{\circ} 55^{\prime} \mathrm{O}, 250 \mathrm{~m}$ de altitud)

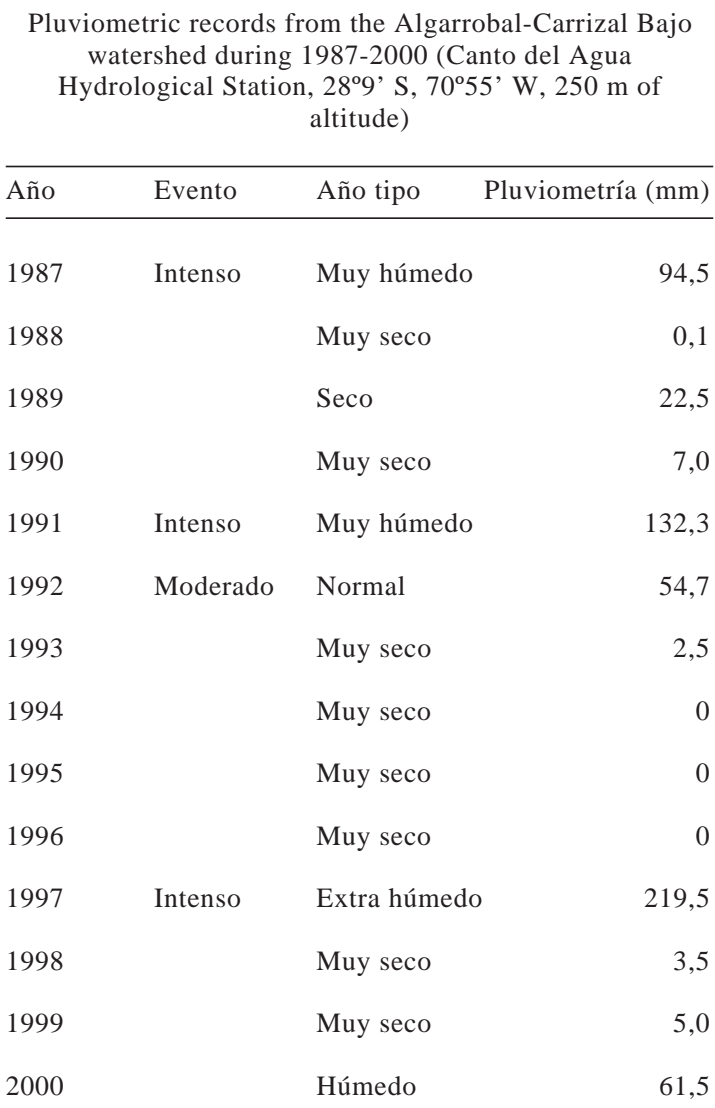

efecto del evento ENOS se reflejó claramente en la contribución numérica de los años: mientras los años no-ENOS seco y no-ENOS húmedo aportaron el 4,9 y el $14,4 \%$, respectivamente, el año ENOS intenso contribuyó con el 80,7 \% de los individuos colectados. Con algunas excepciones (e.g., Psocoptera), el aumento numérico absoluto observado en el año ENOS intenso comparado al año no-ENOS seco, fue mostrado por la mayoría de los taxa. No obstante, la variabilidad numérica fue errática entre los años con mayor pluviometría (años ENOS intenso y no-ENOS húmedo). Mientras seis de los 15 taxa aumentaron sus aportes numéricos en ambos años, ocho lo hicieron solo en el año ENOS intenso; algunos desaparecieron del registro en el año no-ENOS húmedo (e.g., Collembola, Dermaptera, Acarina y Solpugida) y otros mostraron valores inferiores (e.g., Psocoptera) (Tabla 3). Las diferentes condiciones de precipitación se reflejaron igualmente en la composición del ensamble de taxa dominantes $(\geq 3 \%)$. En el año no-ENOS seco y en orden porcentual ascendente el ensamble dominante estuvo constituido por Collembola, Diptera, Psocoptera, Hymenoptera, Acarina y Coleoptera; en el año ENOS intenso el dominio correspondió a Collembola, Coleoptera, Diptera, Lepidoptera, Hymenoptera y Acarina, y en el año no-ENSO húmedo fueron Coleoptera, Diptera e Hymenoptera.

El efecto de la precipitación sobre las relaciones de abundancia de los taxa del ensamble de artrópodos caminadores también se evidenció entre hábitats pedológicos (Fig. 1 y 2). Así, en el año ENOS intenso, mientras Tenebrionidae dominó en el HDC (69,6 \%), Formicidae lo hizo en el HPI $(60,1 \%)$. En el año no-ENOS húmedo, Tenebrionidae dominó en ambos sistemas (89 y 68,9\%, respectivamente). Estas dominancias cambiaron durante los meses de mayor actividad biológica del ecosistema. Bajo el evento ENOS, Formicidae dominó en ambos sistemas en septiembre y diciembre, en octubre, Tenebrionidae dominó en el HDC y Formicidae lo hizo en el HPI (Tabla 4).

Distribución de las abundancias relativas de los tenebriónidos epígeos y efecto del hábitat pedológico

Bajo el evento ENOS, el HDC contribuyó con el $62 \%$ al total de las capturas de los artrópodos epígeos y el HPI lo hizo con el $38 \%$. Tenebrionidae contribuyó, a este total, con el $49,6 \%$ (HDC) y $6,4 \%$ (HPI). Respecto del total de tenebriónidos capturados, el HDC aportó el 92,6 \% y el HPI el 7,4\%. La variabilidad mensual de la denso-actividad de Tenebrionidae fue claramente mayor en el HPI que en el HDC (Tabla 4).

El ensamble de los tenebriónidos caminadores estuvo formado por 12 (HDC) y 10 (HPI) especies. Resultó claro el dominio de dos especies: Gyriosomus kingi Reed y Gyriosomus planicollis Gebien, particularmente en el HDC, donde ambas sumaron el 96,3\% del total 
capturado en el sitio. Esta dominancia se repitió en el HPI, aunque algo disminuida $(61,8 \%)$. Octubre fue el mes que aportó la mayor contribución numérica tanto en el HDC ( 39\%) como en el HPI ( 42\%) (Tabla 5). El ANDEVA de mediciones repetidas reveló que algunas de las diferencias mostradas en la Tabla 5 fueron significativas tanto en la dimensión temporal (i.e., mes de muestreo) como en la espacial (i.e., tipo de hábitat) (Tabla 6).

TABLA 3

Relaciones porcentuales de Arthropoda en el desierto costero transicional de Chile en años con y sin evento ENOS*. Sitio de estudio: Parque Nacional Llanos de Challe. Mes: octubre. $(\mathrm{HDC}=$ hábitat dunario costero)

Percent relationships of Arthropoda inhabiting the transitional coastal desert of Chile in years with and without ENSO events. Study site: Llanos de Challe National Park. Month: October. (HDC = coastal dune habitat)

\begin{tabular}{|c|c|c|c|c|c|c|c|c|c|}
\hline \multirow[t]{3}{*}{ Taxon } & & \multicolumn{6}{|c|}{ Año } & & \\
\hline & & \multicolumn{2}{|c|}{ No-ENOS seco } & \multicolumn{2}{|c|}{ ENOS } & \multicolumn{2}{|c|}{ No-ENOS húmedo } & \multicolumn{2}{|c|}{ Total capturado } \\
\hline & & $\mathrm{n}$ & $\%$ & $\mathrm{n}$ & $\%$ & $\mathrm{n}$ & $\%$ & $\mathrm{n}$ & $\%$ \\
\hline \multirow[t]{11}{*}{ Insecta } & Collembola & 251 & 57,6 & 3023 & 41,4 & 0 & 0 & 3274 & 36,1 \\
\hline & Coleoptera & 16 & 3,6 & 1725 & 23,7 & 964 & 74,2 & 2705 & 29,8 \\
\hline & Diptera & 52 & 12,0 & 679 & 9,3 & 163 & 12,6 & 894 & 9,9 \\
\hline & Lepidoptera & 9 & 1,8 & 666 & 9,2 & 7 & 0,5 & 682 & 7,5 \\
\hline & Hymenoptera & 32 & 7,3 & 399 & 5,5 & 92 & 6,9 & 523 & 5,7 \\
\hline & Homoptera & 5 & 1,1 & 181 & 2,5 & 12 & 0,9 & 198 & 2,2 \\
\hline & Orthoptera & 12 & 2,6 & 151 & 2,0 & 13 & 0,9 & 176 & 1,9 \\
\hline & Dermaptera & 0 & 0 & 109 & 1,4 & 0 & 0 & 109 & 1,2 \\
\hline & Psocoptera & 33 & 7,5 & 9 & $<0,1$ & 2 & 0,1 & 44 & 0,8 \\
\hline & Thysanoptera & 2 & 0,2 & 14 & 0,1 & 25 & 1,9 & 41 & 0,3 \\
\hline & Hemiptera & 2 & 0,3 & 9 & $<0,1$ & 2 & 0,1 & 13 & 0,1 \\
\hline \multirow[t]{4}{*}{ Arachnida } & Acarina & 19 & 4,4 & 253 & 3,5 & 0 & 0 & 272 & 3,0 \\
\hline & Solpugida & 4 & 1,0 & 55 & 0,7 & 0 & 0 & 59 & 0,7 \\
\hline & Araneae & 3 & 0,6 & 31 & 0,4 & 10 & 0,7 & 44 & 0,5 \\
\hline & Scorpionida & 0 & 0 & 18 & 0,2 & 13 & 0,9 & 31 & 0,3 \\
\hline \multicolumn{2}{|l|}{ Total } & 440 & 99,7 & 7322 & 99,9 & 1303 & 99,7 & 9065 & 100 \\
\hline \multicolumn{2}{|c|}{ Porcentaje del total } & 4,9 & & 80,8 & & 14,3 & & 100,0 & \\
\hline
\end{tabular}

* Registro pluviométrico para los años estudiados: 22,5 mm (1989, año no-ENOS seco, 44 \% déficit), 219,5 mm (1997, año ENOS intenso, $443 \%$ superávit), y 61,5 mm (2000, año no-ENOS húmedo, $52 \%$ superávit)

* Rainfall record for the study years: $22.5 \mathrm{~mm}$ (1989, dry no-ENSO year, $44 \%$ below average), $219.5 \mathrm{~mm}$ (1997, intense ENSO year, $443 \%$ above average), y $61.5 \mathrm{~mm}$ (2000, damp no-ENSO year, $52 \%$ above average) 


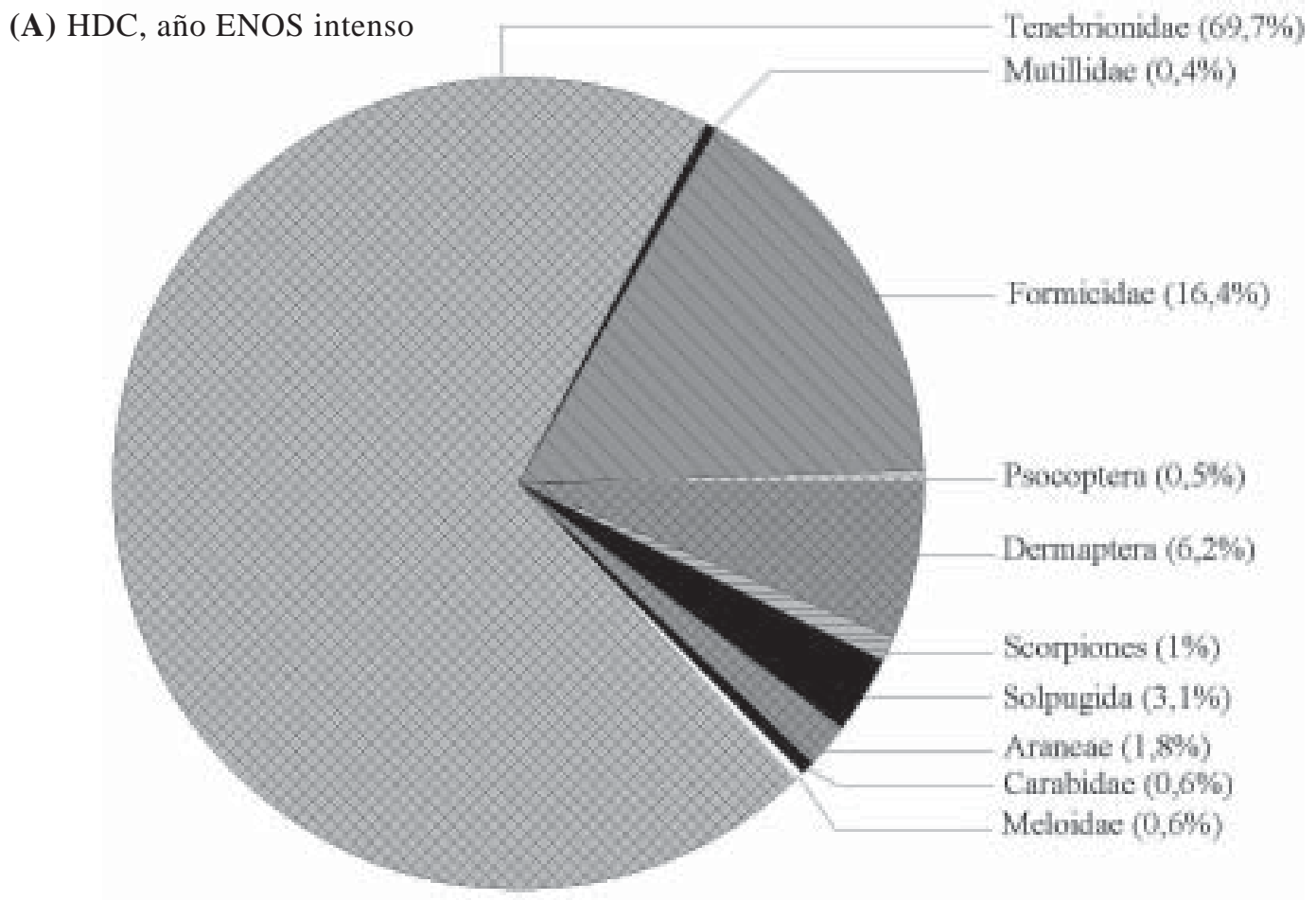

(B) HDC, año no-ENOS húmedo

Tenebrionidae (89\%)

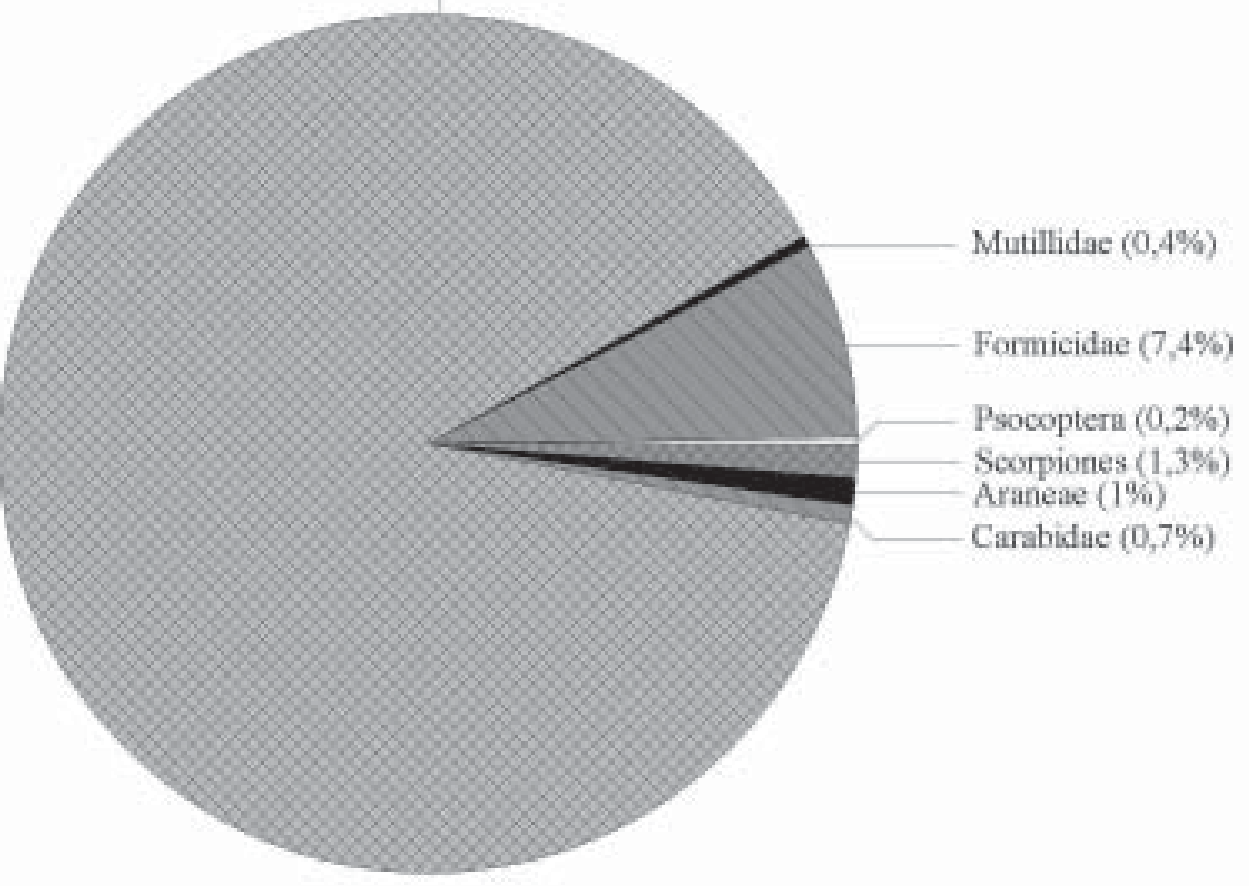

Fig. 1: Relaciones porcentuales de la abundancia relativa de los principales taxa de artrópodos epígeos en el hábitat dunario costero (HDC), del Parque Nacional Llanos de Challe, durante los años ENOS intenso (A) y no-ENOS húmedo (B).

Percent relationships of major taxa of epigean arthropods in the coastal sandy habitat (HDC), at Llanos de Challe National Park, during the years ENSO intense (A) and damp no-ENSO (B). 


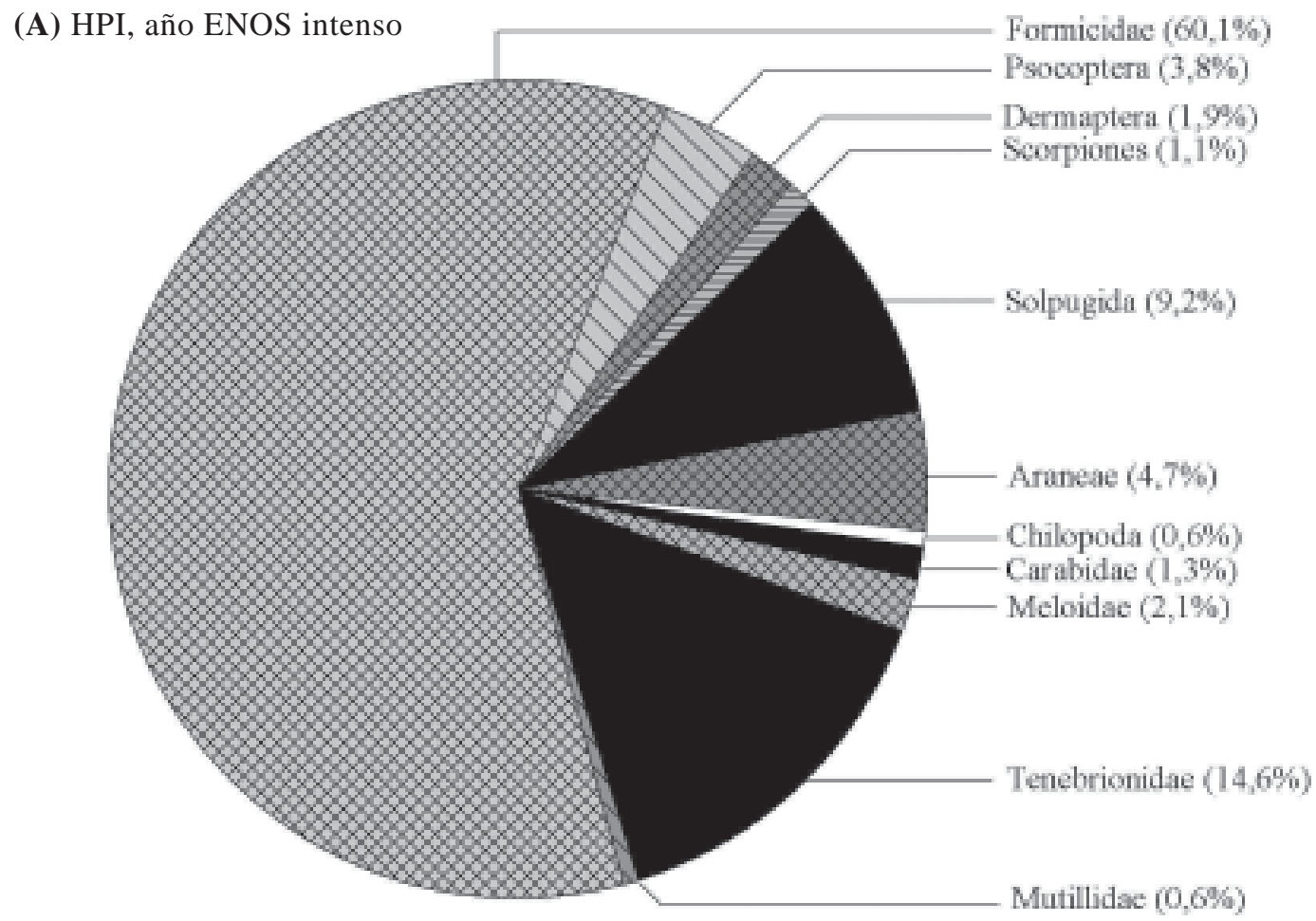

(B) HPI, año no-ENOS húmedo Tenebrionidae $(68,9 \%)$

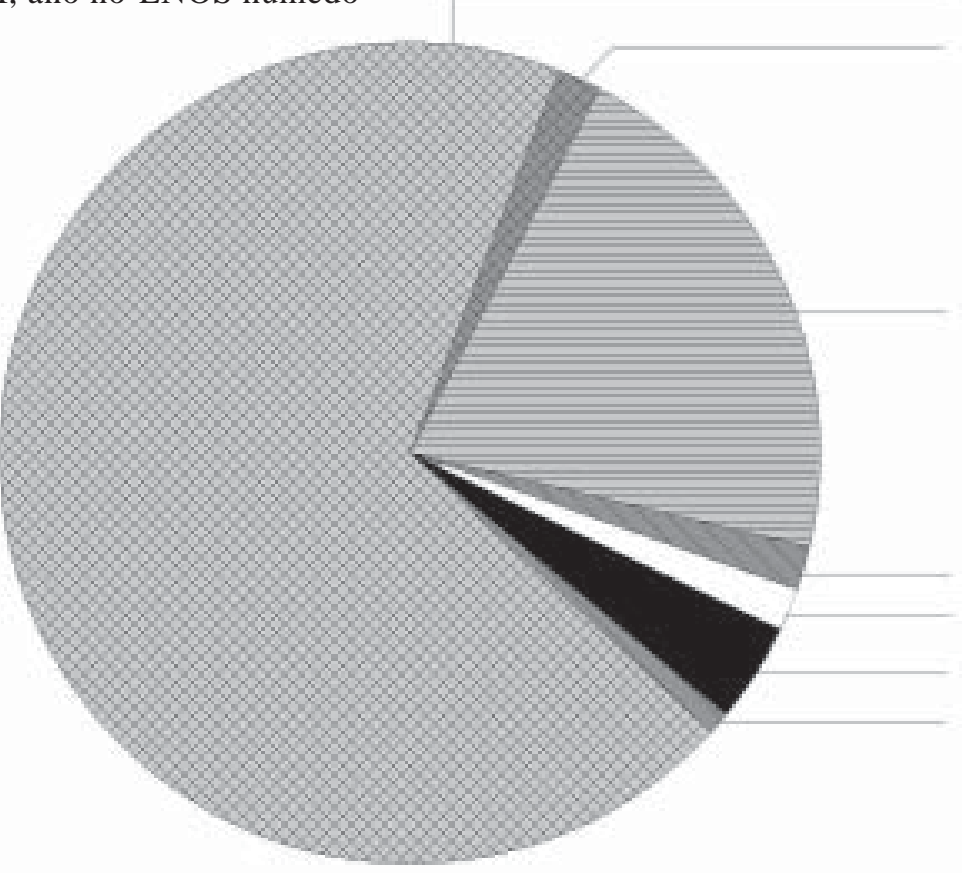

Mutillidae $(1,7 \%)$

Formicidae $\{21 \%)$

Scorpiones $(1,7 \%)$

Solpugida $(1,7 \%)$

Araneac $(3,9 \%)$

Meloidae $(0,9 \%)$

Fig. 2: Relaciones porcentuales de la abundancia relativa de los principales taxa de artrópodos epígeos en el hábitat pedregoso interior (HPI), del Parque Nacional Llanos de Challe, durante los años ENOS intenso (A) y no-ENOS húmedo (B).

Percent relationships of major taxa of epigean arthropods in the inland alluvial habitat (HPI), at Llanos de Challe National Park, during the years ENSO intense (A) and damp no-ENSO (B). 
TABLA 4

Distribución temporal de la relación porcentual de la abundancia de artrópodos caminadores en dos hábitats pedológicos del Parque Nacional Llanos de Challe, en meses de actividad biológica bajo efectos de año ENOS intenso

Temporal distribution of percent relationships of abundance of ground-dwelling arthropods in two pedological habitats present in the Llanos de Challe National Park, in months of biological activity under the effect of intense ENSO year

\begin{tabular}{|c|c|c|c|c|c|c|}
\hline \multirow[t]{3}{*}{ Mes } & \multirow[t]{3}{*}{ Taxon } & & \multicolumn{4}{|c|}{ Hábitat } \\
\hline & & & \multicolumn{2}{|c|}{ HDC } & \multicolumn{2}{|c|}{ HPI } \\
\hline & & & $\mathrm{n}$ & $\%$ & $\mathrm{n}$ & $\%$ \\
\hline \multirow[t]{12}{*}{ Septiembre } & Coleoptera & Carabidae & 9 & 3,3 & 1 & 0,5 \\
\hline & & Meloidae & 3 & 1,1 & 0 & 0 \\
\hline & & Tenebrionidae & 75 & 27,8 & 2 & 1,0 \\
\hline & Hymenoptera & Mutillidae & 1 & 0,3 & 1 & 0,5 \\
\hline & & Formicidae & 150 & 55,7 & 156 & 82,5 \\
\hline & Psocoptera & & 11 & 4,1 & 1 & 0,5 \\
\hline & Dermaptera & & 1 & 0,3 & 5 & 2,5 \\
\hline & Scorpionida & & 0 & 0 & 0 & 0 \\
\hline & Solpugida & & 3 & 1,1 & 11 & 5,8 \\
\hline & Araneae & & 16 & 5,9 & 10 & 5,2 \\
\hline & Chilopoda & & 0 & 0 & 2 & 1,0 \\
\hline & Total & & 269 & 99,6 & 189 & 99,5 \\
\hline \multirow[t]{12}{*}{ Octubre } & Coleoptera & Carabidae & 10 & 0,5 & 8 & 1,2 \\
\hline & & Meloidae & 6 & 0,3 & 13 & 2,0 \\
\hline & & Tenebrionidae & 1.229 & 69,6 & 92 & 14,5 \\
\hline & Hymenoptera & Mutillidae & 8 & 0,4 & 4 & 0,6 \\
\hline & & Formicidae & 289 & 16,3 & 379 & 60,1 \\
\hline & Psocoptera & & 9 & 0,5 & 24 & 3,8 \\
\hline & Dermaptera & & 109 & 6,1 & 12 & 1,9 \\
\hline & Scorpionida & & 18 & 1,0 & 7 & 1,1 \\
\hline & Solpugida & & 55 & 3,1 & 58 & 9,1 \\
\hline & Araneae & & 31 & 1,7 & 30 & 4,7 \\
\hline & Chilopoda & & 0 & 0 & 4 & 0,6 \\
\hline & Total & & 1.764 & 99,5 & 631 & 99,6 \\
\hline \multirow[t]{13}{*}{ Diciembre } & Coleoptera & Carabidae & 126 & 9,9 & 5 & 0,4 \\
\hline & & Meloidae & 2 & 0,1 & 2 & 0,1 \\
\hline & & Tenebrionidae & 337 & 26,4 & 37 & 3,0 \\
\hline & Hymenoptera & Mutillidae & 63 & 4,9 & 9 & 0,7 \\
\hline & & Formicidae & 565 & 44,3 & 934 & 76,8 \\
\hline & Psocoptera & & 19 & 1,5 & 6 & 0,5 \\
\hline & Dermaptera & & 0 & 0 & 39 & 3,2 \\
\hline & Scorpionida & & 32 & 2,5 & 9 & 0,7 \\
\hline & Solpugida & & 69 & 5,4 & 100 & 8,2 \\
\hline & Araneae & & 62 & 4,8 & 74 & 6,1 \\
\hline & Chilopoda & & 0 & 0 & 0 & 0 \\
\hline & Total & & 1.275 & 99,8 & 1.215 & 99,7 \\
\hline & Total & & 3.308 & 100,0 & 2.035 & 100,0 \\
\hline
\end{tabular}

HDC = hábitat dunario costero; HPI = hábitat pedregoso interior HDC $=$ coastal dune habitat HPI = inland stony-coarse habitat 
TABLA 5

Relaciones porcentuales temporales de los tenebriónidos epígeos presente en dos hábitats pedológicos del Parque Nacional Llanos de Challe, durante año ENOS intenso

Temporal percent relationships of epigean tenebrionids inhabiting two pedological habitats of Llanos de Challe National Park, during intense ENSO year

\begin{tabular}{|c|c|c|c|c|c|c|c|}
\hline \multirow[t]{3}{*}{ Mes } & \multirow[t]{3}{*}{ Especie } & \multicolumn{4}{|c|}{ Hábitat } & \multicolumn{2}{|c|}{ Total mes } \\
\hline & & \multicolumn{2}{|c|}{ HDC } & \multicolumn{2}{|c|}{ HPI } & & \\
\hline & & $\mathrm{n}$ & $\%$ & $\mathrm{n}$ & $\%$ & $\mathrm{n}$ & $\%$ \\
\hline \multirow[t]{13}{*}{ Septiembre } & Gyriosomus kingi Reed & 54 & 72,0 & 1 & 50,0 & 55 & 71,4 \\
\hline & Gyriosomus planicollis Geb. & 10 & 13,3 & 0 & 0 & 10 & 12,9 \\
\hline & Gyriosomus parvus Sol. & 0 & 0 & 1 & 50,0 & 1 & 1,3 \\
\hline & Gyriosomus curtisi Fair. & 0 & 0 & 0 & 0 & 0 & 0 \\
\hline & Praocis sp. & 1 & 1,3 & 0 & 0 & 1 & 1,3 \\
\hline & Scotobius sp. & 7 & 9,3 & 0 & 0 & 7 & 9,0 \\
\hline & Thinobatis sp. & 2 & 2,7 & 0 & 0 & 2 & 2,6 \\
\hline & Nycterinus sp. & 0 & 0 & 0 & 0 & 0 & 0 \\
\hline & Psammetichus sp. & 0 & 0 & 0 & 0 & 0 & 0 \\
\hline & Geoborus sp. & 0 & 0 & 0 & 0 & 0 & 0 \\
\hline & Entomochilus sp. & 0 & 0 & 0 & 0 & 0 & 0 \\
\hline & Psectrascelis sp. & 1 & 1,3 & 0 & 0 & 1 & 1,3 \\
\hline & Total & 75 & 99,9 & 2 & 100 & 77 & 99,8 \\
\hline \multirow[t]{13}{*}{ Octubre } & Gyriosomus kingi Reed & 878 & 71,5 & 60 & 65,2 & 939 & 71,1 \\
\hline & Gyriosomus planicollis Geb. & 321 & 26,1 & 15 & 16,3 & 336 & 25,4 \\
\hline & Gyriosomus parvus Sol. & 0 & 0 & 1 & 1,0 & 0 & 0 \\
\hline & Gyriosomus curtisi Fair. & 0 & 0 & 8 & 8,7 & 8 & 0,6 \\
\hline & Praocis sp. & 1 & 0,08 & 1 & 1,1 & 2 & 0,1 \\
\hline & Scotobius sp. & 3 & 0,2 & 1 & 1,1 & 4 & 0,3 \\
\hline & Thinobatis sp. & 2 & 0,1 & 1 & 1,1 & 3 & 0,2 \\
\hline & Nycterinus sp. & 3 & 0,2 & 0 & 0 & 3 & 0,2 \\
\hline & Psammetichus sp. & 9 & 0,7 & 3 & 3,2 & 12 & 0,9 \\
\hline & Geoborus sp. & 8 & 0,6 & 2 & 2,1 & 10 & 0,8 \\
\hline & Entomochilus sp. & 1 & 0,08 & 0 & 0 & 1 & $<0,1$ \\
\hline & Psectrascelis sp. & 3 & 0,2 & 0 & 0 & 3 & 0,2 \\
\hline & Total & 1.229 & 99,7 & 2 & 99,9 & 1.321 & 99,9 \\
\hline \multirow[t]{14}{*}{ Diciembre } & Gyriosomus kingi Reed & 307 & 91,1 & 0 & 0 & 307 & 82,0 \\
\hline & Gyriosomus planicollis Geb. & 10 & 2,9 & 5 & 13,5 & 15 & 4,0 \\
\hline & Gyriosomus parvus Sol. & 0 & 0 & 1 & 2,7 & 0 & 0 \\
\hline & Gyriosomus curtisi Fair. & 0 & 0 & 13 & 35,1 & 14 & 3,7 \\
\hline & Praocis sp. & 8 & 2,3 & 7 & 19,0 & 15 & 4,0 \\
\hline & Scotobius sp. & 3 & 0,9 & 0 & 0 & 3 & 0,8 \\
\hline & Thinobatis sp. & 4 & 1,1 & 0 & 0 & 4 & 1,0 \\
\hline & Nycterinus sp. & 1 & 0,2 & 0 & 0 & 1 & 0,3 \\
\hline & Psammetichus sp. & 2 & 0,5 & 7 & 19,0 & 9 & 2,4 \\
\hline & Geoborus sp. & 2 & 0,5 & 0 & 0 & 2 & 0,5 \\
\hline & Entomochilus sp. & 0 & 0 & 4 & 10,7 & 4 & 1,0 \\
\hline & Psectrascelis sp. & 0 & 0 & 0 & 0 & 0 & 0 \\
\hline & Total & 337 & 99,5 & 37 & 100,0 & 374 & 99,7 \\
\hline & Total período & 1.641 & 100,0 & 131 & 100,0 & 1.772 & 100,0 \\
\hline
\end{tabular}

HDC = hábitat dunario costero; HPI = hábitat pedregoso interior HDC $=$ coastal dune habitat HPI $=$ inland stony-coarse habitat 
TABLA 6

Valores de F del ANDEVA para efecto del mes de captura y tipo de hábitat sobre la denso-actividad de los tenebriónidos epígeos de la estepa costera desértico transicional de Chile, durante año ENOS intenso. Sitio de estudio: Parque Nacional Llanos de Challe

\footnotetext{
F-values of the ANOVA test to evaluate effect of sampling month and habitat type on the denso-activity of epigean tenebrionids inhabiting the coastal steppe of the transitional desert of Chile, intense ENSO year. Study site: Llanos de Challe National Park
}

\begin{tabular}{|c|c|c|c|c|}
\hline Taxon & $\mathrm{F}_{\text {Tiempo }}$ & Valor de P & $\mathrm{F}_{\text {Hábitat }}$ & Valor de $\mathrm{P}$ \\
\hline Tenebrionidae in toto & $3,82 *$ & 0,046 & $7,00 * *$ & 0,025 \\
\hline Gyriosomus spp. & $4,82 *$ & 0,034 & $8,63 * *$ & 0,015 \\
\hline G. kingi & $3,53 *$ & 0,052 & $7,85^{* *}$ & 0,019 \\
\hline G. planicollis & $18,07 * *$ & 0,005 & $17,96^{*}$ & 0,002 \\
\hline G. parvus & 1,00 & 0,402 & 1,00 & 0,340 \\
\hline G. curtisi & $15,29 * *$ & $<0,001$ & $57,65 * *$ & $<0,001$ \\
\hline Praocis spp. & 1,62 & 0,246 & 0,64 & 0,442 \\
\hline P. chilensis & 1,00 & 0,402 & 1,00 & 0,340 \\
\hline Praocis sp. & 3,23 & 0,083 & 1,11 & 0,318 \\
\hline Scotobius spp. & 1,00 & 0,402 & $11,08 * *$ & 0,008 \\
\hline Thinobatis spp. & 1,25 & 0,333 & $20,42 * *$ & 0,001 \\
\hline Nycterinus spp. & 1,52 & 0,265 & 3,48 & 0,092 \\
\hline Psammetichus spp. & $5,09 *$ & 0,030 & 1,17 & 0,305 \\
\hline Geoborus spp. & 2,58 & 0,125 & 2,12 & 0,175 \\
\hline Entomochilus spp. & 0,45 & 0,650 & 0,45 & 0,650 \\
\hline
\end{tabular}

${ }^{1}$ Grados de libertad para efecto del mes = 2; grados de libertad para efecto del tipo de hábitat =1; grados de libertad en el denominador $=12$

${ }^{1}$ Degrees of freedom for the month effect $=2$; degrees of freedom for habitat effect $=1$; degrees of freedom in the denominator $=12$

\section{DISCUSIÓN}

Estructura taxonómica del ensamble de Arthropoda

A pesar que el método de captura no es el más adecuado para los grupos de insectos voladores (Work et al. 2002), la presencia porcentual significativa de Diptera y Lepidoptera encontrada en este estudio concuerda con resultados similares hallados en trabajos previos (Cepeda-Pizarro et al. en prensa). Considerando que el área de estudio constituye un foco de endemismo y diversidad florística (Armesto \& Vidiella 1993, Armesto et al. 1993, Vidiella et al. 1999), la presencia de estos taxa enfatiza la necesidad de abordar análisis taxonómicos y funcionales acabados, particularmente por su probable condición de elementos endémicos y agentes polinizadores.

El estudio también confirma observaciones previas respecto de la dominancia numérica que pueden lograr algunos taxa de artrópodos edáficos (e.g., colémbolos y psocópteros) y caminadores (e.g., ácaros, formícidos y tenebriónidos) en los ecosistemas desérticos costeros chilenos (Cepeda-Pizarro et al. en prensa). A diferencia del nivel de conocimiento que se tiene respecto de las causas de las irrupciones poblacionales de micromamíferos del desierto transicional del Chile (Péfaur et al. 1979, Meserve et al. 1995, 2003, Lima et al. 1999, Yunger et al. 2002), los incrementos demográficos observados en grupos de Arthropoda no han sido a la fecha examinados en detalle (Moroni 1972, Fuentes \& 
Campusano 1985, Cepeda-Pizarro \& Vásquez $1999^{1}$, Cepeda-Pizarro et al. $1996^{2}, 2000^{3}$, 2003). Los incrementos poblacionales de ácaros registrados en este trabajo correspondieron a miembros del ensamble Parasitengonae (Krantz 1978), Balaustium spp. (M. Casanueva, comunicación personal). Irrupciones poblacionales similares han sido observadas en el desierto de Arizona (Newell \& Tevis 1960). Aun cuando no se hizo un análisis taxonómico específico para los formícidos, las especies más abundantes fueron Solenopsis latastei Emery y Brachymymex giardii Emery. El conocimiento taxonómico y ecológico de la fauna mirmecológica del desierto árido chileno es incipiente (Snelling \& Hunt 1975, Fuentes et al. 1996, Torres-Contreras 2001). En este trabajo se documenta, de manera preliminar, el papel que juegan la precipitación y el sustrato pedológico en la abundancia de estos insectos. Nuestros resultados concuerdan con Schumacher \& Whitford (1976), Whitford (1978) y Maes et al. (2003), en que existen especies que muestran diferencias en la actividad estacional, debido posiblemente a interacciones de competencia entre las comunidades de estos insectos. Puesto que la actividad de la fauna mirmecológica del ambiente desértico es controlada por diversos factores (e.g., precipitaciones, productividad primaria) (Whitford 1978, 1999, Whitford et al. 1980, Marsh 1988), las interacciones de estos factores, al variar entre años y estacionalmente, hacen que la estructura observada del ensamble cambie constantemente (Whitford 1978, Heatwole 1996, 2001), aspecto no evaluado en la fauna mirmecológica local.

La abundancia y diversidad de Tenebrionidae observada en este estudio concuerda con lo encontrado en otros ecosistemas áridos, por ejemplo de Arizona

\footnotetext{
1 CEPEDA-PIZARRO J \& H VÁSQUEZ (1999) Observaciones sobre una irrupción poblacional de Elasmoderus wagenknechti (Orthoptera: Tristiridae). XXI Congreso Nacional de Entomología, Arica, Chile. Libro de Resúmenes: 14

2 CEPEDA-PIZARRO J, H VÁSQUEZ \& G ARANCIO (1996) Asociación recurso floral/artrópodos en una comunidad desértica del norte de Chile. XVIII Congreso Nacional de Entomología. Temuco, Chile, Libro de Resúmenes: 28

${ }^{3}$ CEPEDA-PIZARRO J, S VEGA \& J PIZARRO (2000) Irrupciones poblacionales de Elasmoderus wagenknechti (Orthoptera: Tristiridae). XXII Congreso Nacional de Entomología, Valdivia, Chile. Libro de Resúmenes: 27.
}

(Ahearn 1971), Tenerife (de los Santos et al. 2000, 2002), Atacama y Namibia (Crawford et al. 1993), Negev (Ayal \& Merkl 1994, Krasnov et al. 1996), Texas (Deslippe et al. 2001), Sinaí (Semida et al. 2001), Sechura (Giraldo \& Arellano 2003). Se considera que estos insectos desempeñan un papel clave en los procesos de fragmentación biológica del recurso vegetal, en los ciclos de los nutrientes y en la dieta de otros organismos consumidores, particularmente vertebrados (Wallwork 1982, Dajoz 1984, Sheldon \& Rogers 1984, Wise 1985, Díaz \& Díaz 1990, Mikhail 1993, Flores 1998, Fattorini 2001, Semida et al. 2001). Junto a lo anterior, algunas especies de Tenebrionidae se consideran posibles indicadores de las condiciones climáticas (Marcuzzi 1951). El área estudiada, particularmente la franja arenosa costera constituye un foco de endemismo y diversidad de Tenebrionidae (Pizarro-Araya \& Jerez 2004, Pizarro-Araya et al. 20044), siendo evidente la dominancia numérica y diversidad específica del género Gyriosomus, características que sugieren para este grupo de Pimeliinae una probable importancia local como recurso trófico y dinamizador del ciclo de nutrientes (PizarroAraya \& Flores 2004).

Distribución de las abundancias relativas de Arthropoda y efecto de la precipitación

Shmida et al. (1985) han señalado que el funcionamiento y la estructura de los ecosistemas áridos descansan sobre la complementariedad entre las fases áridas y húmedas que oscilatoriamente experimenta el sistema (ver Tabla 3). Mientras la etapa árida es la fase característica, dominante y predecible del sistema, la etapa húmeda es un momento breve, ocasional e impredecible. De acuerdo a estos autores, ciertas especies poseerían las estrategias ecológicas requeridas para obtener provecho de los recursos disponibles presentes en la fase árida, aunque estos sean escasos y de baja calidad. Del mismo modo, otro conjunto de especies tendría las estrategias ecológicas necesarias para aprovechar los recursos

${ }^{4}$ PIZARRO-ARAYA J, V JEREZ \& J CEPEDA-PIZARRO (2004) Patrones distribucionales y áreas de endemismo para coleópteros epígeos del desierto costero del norte de Chile $\left(25^{\circ}-32^{\circ}\right.$ Lat S). XXVI Congreso Nacional de Entomología, Concepción, Chile. Libro de Resúmenes: 17. 
disponibles en la fase húmeda, esta vez más abundantes y mejores en calidad. En relación a los artrópodos y según observaciones realizadas en ecosistemas desérticos insulares (Polis et al. 1997), los elementos fitófagos predominan en los años de precipitación elevada y los elementos detritívoros y carroñeros lo hacen en los años secos. La disminución o ausencia de actividad biológica de Arthropoda durante los períodos de escasez de recursos y/o elevado déficits hídrico constituyen respuestas adaptativas desplegadas por estos organismos en un ambiente donde la expresión de las condiciones favorables tiene baja predictibilidad (Whitford 1978), particularmente si el ambiente está sujeto a las incursiones del evento ENOS (Romero \& Garrido 1985, Jaksic 1998).

Diversos autores (Péfaur et al. 1979, Meserve et al. 1995, 2003, Jaksic 1998, 2001, Lima et al. 1999, Gutiérrez et al. 2000a, 2000b, Holmgreen et al. 2001, Yunger et al. 2002, Gutiérrez \& Meserve 2003, Kelt et al. 2004a, 2004b) han llamado la atención acerca del efecto ecológico que el evento ENOS tiene sobre el ecosistema árido y su relación con la ocurrencia de la fase húmeda del desierto transicional de Chile (Romero \& Garrido 1985, Jaksic 1998). El año ENOS intenso, considerado el fenómeno climático del siglo por su intensidad (Santibáñez \& Uribe 1999, Changton 2000, Deslippe et al. 2001), no solo cambió la abundancia de la mayoría de los taxa del área, algunos de los cuales aumentaron su abundancia en varios órdenes de magnitudsino que también cambió la composición del ensamble de taxa dominantes. En concordancia con Polis et al. (1997), aumentó la presencia de artrópodos fitófagos (e.g., Lepidoptera, Hemiptera, Orthoptera, Thysanoptera), elementos depredadores (e.g., Solpugida, Scorpionida, Araneae) y carroñeros u omnívoros (e.g., Dermaptera, Tenebrionidae, Formicidae). En parcial concordancia con Polis et al. (1997), durante el año seco del período estudiado, aun cuando los ejemplares capturados fueron relativamente bajos, el ensamble de artrópodos estuvo dominado por Collembola, Diptera, Formicidae, Psocoptera y Acarina. A dos años de ocurrencia del año ENOS intenso, el registro del año no-ENOS húmedo mostró dominancia de Tenebrionidae, Diptera y Formicidae. Sorpresivamente,
Collembola, un grupo indicador de condiciones favorables de humedad edáfica (Butcher et al. 1971) y abundante en el área en los años noENOS seco y ENOS intenso, no fue colectado en la campaña del año no-ENOS húmedo. Este resultado amerita un análisis más acabado de la distribución y respuestas numéricas de este grupo edáfico.

Según lo han documentado Deslippe et al. (2001) con Eleodes extricata en Texas, es posible que algunos aspectos de la biología y ecología de las especies locales de Tenebrionidae estén muy ligados a la ocurrencia y características del evento ENOS. En el presente estudio mostramos que Tenebrionidae respondió a ambos pulsos de humedad, incrementando su número en varios órdenes de magnitud respecto del año seco. Las respuestas numéricas de algunos taxa (e.g., Tenebrionidae, Formicidae) estuvieron mediadas por las características del hábitat pedológico. El efecto del hábitat sobre la distribución y abundancia de Tenebrionidae está ampliamente documentado en la literatura (Calkins \& Kirk 1975, Thomas 1979, Sheldon \& Rogers 1984, Parmenter et al. 1989a, 1989b, Stapp 1997, Carpaneto \& Fattorini 2001, 2003, Deslippe et al. 2001, de los Santos et al. 2002, Giraldo \& Arellano 2003).

Distribución de las abundancias relativas de los tenebriónidos epígeos y efecto del hábitat pedológico

La preferencia de Tenebrionidae por el hábitat arenoso fue clara. Esta preferencia puede estar determinada por diferentes factores. Por un lado, un hábitat arenoso permitiría realizar las oviposturas más profundamente y con menos esfuerzo energético, ahorro que puede destinarse a la producción de huevos o a buscar microhábitats cuya humedad edáfica pueda reducir la probabilidad de las larvas de morir por deshidratación (Rogers et al. 1998, Deslippe et al. 2001). Por otro lado favorecería un adecuado suministro de alimentos (e.g., presencia de raíces efímeras en descomposición por hongos, alimentos de alto valor nutritivo) (Seely 1979, Slobodchikoff 1983).

En conclusión, el efecto del evento ENOS se reflejó claramente en un incremento numérico en la mayoría de los taxa registrados. Aunque más sutilmente, este efecto también se reflejó en la 
composición del ensamble de taxa dominantes y entre hábitats pedológicos, particularmente con Tenebrionidae y Formicidae. Especialmente en las dunas costeras, Tenebrionidae dominó claramente el ensamble de artrópodos epígeos del Parque Nacional Llanos de Challe, siendo Gyriosomus Guérin-Méneville el género más diverso y abundante.

\section{AGRADECIMIENTOS}

Agradecemos a Corporación Nacional Forestal (III Región) por otorgar las facilidades para trabajar en el PN Llanos de Challe y a Gina Arancio, curadora del herbario (Universidad de La Serena), por la descripción florística de los sitios de estudio. Extendemos nuestros agradecimientos a Julio R. Gutiérrez (Universidad de La Serena), Viviane Jerez (Universidad de Concepción), y dos revisores anónimos por los comentarios y sugerencias realizadas a la versión anterior del manuscrito. El financiamiento fue proporcionado por la Universidad de La Serena (Dirección de Investigación, DIULS), subprograma ecosistemas áridos y semiáridos.

\section{LITERATURA CITADA}

AHEARN GA (1971) Ecological factors affecting population sampling of desert tenebrionid beetles. American Midland Naturalist 86: 385-406.

ANÓNIMO (1996) Statistix for windows. User's manual. Analytical software. Tallahassee, Florida, USA. v + 333 pp.

ARMESTO JJ \& PE VIDIELLA (1993) Plant life forms and biogeographic relations of the flora of Lagunillas $\left(30^{\circ} \mathrm{S}\right)$ in the fog-free pacific coastal desert. Annals of the Missouri Botanical Garden 80: 499-511.

ARMESTO JJ, PE VIDIELLA \& JR GUTIÉRREZ (1993) Plant communities of the fog-free coastal desert of Chile: plant strategies in a fluctuating environment. Revista Chilena de Historia Natural 66: 271-282.

ARTIGAS JN (1994) Entomología económica. Ediciones Universidad de Concepción. Concepción, Chile. Volumen 1: v + 1126 pp.; Volumen 2: v + 943 pp.

AYAL Y \& O MERKL (1994) Spatial and temporal distribution of tenebrionid species (Coleoptera) in the Negev highlands, Israel. Journal of Arid Environments 27: 347-361.

BUTCHER JW, R SNIDER \& RJ SNIDER (1971) Bioecology of edaphic Collembola and Acarina. Annual Review of Entomology 16: 249-288.

CALKINS CO \& VM KIRK (1975) Distribution of false wireworms (Coleoptera: Tenebrionidae) in relation to soil texture. Environmental Entomology 4: 373374.
CARPANETO GM \& S FATTORINI (2001) Spatial and seasonal organisation of a darkling beetle (Coleoptera, Tenebrionidae) community inhabiting a Mediterranean coastal dune system. Italian Journal of Zoology 68: 207-214.

CARPANETO GM \& S FATTORINI (2003) Seasonal occurrence and habitat distribution of tenebrionid beetles inhabiting a Mediterranean coastal dune (Circeo National Park, Italy). Revue d'Ecologie-La Terre et la Vie 58: 293-306.

CEPEDA-PIZARRO J, S VEGA, H VÁSQUEZ \& $M$ ELGUETA (2003) Morfometría y dimorfismo sexual de Elasmoderus wagenknechti (Libermann) (Orthoptera: Tristiridae) en dos eventos de irrupción poblacional. Revista Chilena de Historia Natural 76: 417-435.

CEPEDA-PIZARRO J, J PIZARRO-ARAYA \& $\mathrm{H}$ VÁSQUEZ (2005) Variación en la abundancia de Arthropoda en un transecto latitudinal del desierto costero transicional de Chile, con énfasis en los tenebriónidos epígeos. Revista Chilena de Historia Natural 78: 651-663.

CHANGTON SA (2000) El Niño 1997-1998: the climate Event of the Century. Oxford University Press, New York, New York, USA. xii +215 pp.

CIGLIANO MM, WP KEMP \& T KALARIS (1995) Spatio-temporal characteristics of rangeland grasshopper (Orthoptera: Acrididae) regional outbreaks in Montana. Journal of Orthoptera Research (USA) 4: 111-126.

COWLING RM, PW RUNDEL, BB LAMONT, MK ARROYO \& M ARIANOUTSOU (1996) Plant diversity in Mediterranean-climate regions. Trends in Ecology and Evolution 11: 362-366.

CRAWFORD CS, WP MACKAY \& JG CEPEDAPIZARRO (1993) Detritivores of the Chilean arid zone $\left(27-32^{\circ} \mathrm{S}\right)$ and the Namib Desert: a preliminary comparison. Revista Chilena de Historia Natural 66: 283-289.

DAJOZ R (1984) Les coleópteres Ténebrionides des deserts. Bulletin des Naturalistes Parisiens (France), Nouvelle Serie 38: 26-57.

DE LOS SANTOS A, JP de NICOLAS \& F FERRER (2002) Habitat selection and assemblage structure of darkling beetles (Col. Tenebrionidae) along environmental gradients on the Island of Tenerife (Canary Islands). Journal of Arid Environments 52: 63-85.

DE LOS SANTOS A, LA GÓMEZ-GONZÁLEZ, C ALONSO, CD ARBELO \& JP de NICOLÁS (2000) Adaptative trends of darkling beetles (Col. Tenebrionidae) on environmental gradients on the island of Tenerife (Canary Islands). Journal of Arid Environments 45: 85-98.

DESLIPPE RJ, JR SALAZAR \& YL GUO (2001) A darkling beetle population in West Texas during the 1997-1998 El Niño. Journal of Arid Environments 49: 711-721.

DÍAZ JA \& M DÍAZ (1990) Estimas de tamaños y biomasas de artrópodos aplicables al estudio de la alimentación de vertebrados insectívoros. Doñana, Acta Vertebrados (España) 17: 67-74.

FATTORINI S (2001) Temporal and spatial variations in darkling beetle predation by kestrels and other raptors in a Mediterranean urban area. Biologia 56: 165-170.

FLORES GE (1997) Revisión de la tribu Nycteliini (Coleoptera: Tenebrionidae). Revista de la Sociedad Entomológica Argentina 56: 1-19.

FLORES GE (1998) Tenebrionidae. En: Morrone JJ \& S Coscarón (eds) Biodiversidad de artrópodos 
argentinos: una perspectiva biotaxonómica: 232257. Ediciones Sur, Buenos Aires, Argentina.

FUENTES E \& C CAMPUSANO (1985) Pest outbreaks and rainfall in the semiarid-region of Chile. Journal of Arid Environments 8: 67-72.

FUENTES JE, S HERRERA \& RG MEDEL (1996) Observaciones preliminares sobre uso de recursos y temperatura de actividad en ensambles de hormigas granívoras del norte de Chile. Acta Entomológica Chilena 20: 13-17.

GAJARDO R (1993) La vegetación natural de Chile. Editorial Universitaria, Santiago, Chile. 165 pp.

GASTON KJ (2000) Global patterns in biodiversity. Nature 405: 220-227.

GIRALDO A \& G ARELLANO (2003) Resiliencia de la comunidad epígea de Coleoptera en las Lomas de Lachay después del evento el Niño 1997-98. Ecología Aplicada 2: 59-68.

GIRALDO A, D PÉREZ \& G ARELLANO (2004) Respuesta de la comunidad de arañas epígeas (Araneae) en las "Lomas de Lachay", Perú, ante la ocurrencia del Evento El Niño 1997-98. Ecología Aplicada 3: 45-58

GUTIÉRREZ JR \& PL MESERVE (2003) El Niño effects on soil seed bank dynamics in north-central Chile. Oecologia 134: 511-517.

GUTIÉRREZ JR, G ARANCIO \& FM JAKSIC (2000a) Variation in vegetation and seed bank in a Chilean semi-arid community affected by ENSO 1997. Journal of Vegetation Science 11: 641-648.

GUTIÉRREZ JR, PL MESERVE \& FM JAKSIC (2000b) Efectos de la Corriente de El Niño sobre la biota terrestre de ecosistemas áridos de Sudamérica. En: Jiménez-Milón P, C Talavera-Delgado, L VillegasParedes, A Ortega-Paredes \& F Villasante-Benavides (eds) Memorias del IV congreso latinoamericano de ecología. ecología y desarrollo sostenible: reto de la América Latina para el tercer milenio: 91-94. Industria Gráfica Regentus, Arequipa, Perú.

HEATWOLE H (1996) Ant assemblages at their dry limits: the northern Atacama desert, Peru, and the Chott El Djerid, Tunisia. Journal of Arid Environments 33: 449-456.

HEATWOLE H (2001) Realized Ant assemblages in the Namib, Kalahari and Kara-Kum deserts. En: Prakash I (ed) Ecology of desert environments: 301-332. Scientific Publishers, Jodhpur, India.

HOLMGREEN M, M SCHEFFER, E EXCURRA, JR GUTIÉRREZ \& GMJ MOHREN (2001) El Niño effects on the dynamics of terrestrial ecosystems. Trends in Ecology and Evolution 16: 89-94.

JAKSIC FM (1998) The multiple facets of El Niño/ Southern Oscillation in Chile. Revista Chilena de Historia Natural 71: 121-131.

JAKSIC FM (2001) Ecological effects of El Niño in terrestrial ecosystems of western South America. Ecography 24: 241-250.

KELT DA, PL MESERVE \& JR GUTIÉRREZ (2004a) Seed removal by small mammals, birds and ants in semi-arid Chile, and comparison with other systems. Journal of Biogeography 31: 931-942.

KELT DA, PL MESERVE, LK NABORS, ML FORISTER \& JR GUTIÉRREZ (2004b) Foraging ecology of small mammals in semiarid Chile: the interplay of biotic and abiotic effects. Ecology 85: 383-397.

KRANTZ GW (1978) A manual of acaralogy. Second edition. Oregon State University Book Stores , Inc., Corvallis, Oregon, USA. 509 pp.

KRASNOV B, D WARD \& G SHENBROT (1996) Body size and length variation in several species of darkling beetles (Coleoptera: Tenebrionidae) along a rainfall and altitudinal gradient in the Negev desert (Israel). Journal of Arid Environments 34: 477-489.

KULZER H (1954) Neunter Beitrag zur Kenntnis der Tenebrioniden (Col.) Eine Studie über die Tribus Nycteliini. Entomologische Arbeiten aus dem Museum George Frey (Alemania) 5: 14-267.

KULZER H (1959) Neue Tenebrioniden aus Südamerika (Col.) 18 Beitrag zur Kenntnis der Tenebrioniden. I Die Gattung Gyriosomus Guérin. (Nycteliini). Entomologische Arbeiten aus dem Museum George Frey (Alemania) 10: 523-547.

LIMA M, PA MARQUET \& FM JAKSIC (1999) El Niño events, precipitation patterns, and rodent outbreaks are statistically associated in semiarid Chile. Ecography 22: 213-218.

MAES D, H VAN DYCK, W VANREUSEL \& J CORTENS (2003) Ant communities (Hymenoptera: Formicidae) of Flemish (north Belgium) wet heathlands, a declining habitat in Europe. European Journal of Entomology 100: 545-555.

MARCUZZI G (1951) Tenebrioni como indicatore del clima. Revista di biologia di Perugia (Italy) 43: 399-437.

MARSH AC (1988) Activity patterns of some Namib desert ant. Journal of Arid Environments 14: 61-73.

MARTICORENA C, FA SQUEO, G ARANCIO \& M MUÑOZ (2001) Catálogo de la flora vascular de la IV Región de Coquimbo. En: Squeo FA, G Arancio \& JR Gutiérrez (eds) Libro rojo de la flora nativa y de los sitios prioritarios para su conservación: Región de Coquimbo: 105-142. Ediciones Universidad de La Serena, La Serena, Chile.

MESERVE PL, DA KELT, WB MILSTEAD \& JR GUTIÉRREZ (2003) Thirteen years of shifting topdown and bottom-up control. BioScience 53: 633-646.

MESERVE PL, JA YUNGER, JR GUTIÉRREZ, LC CONTRERAS, WB MILSTEAD, BK LANG, KL CRAMER, S HERERRA, VO LAGOS, SI SILVA, ET TABILO, MA TORREALBA \& FM JAKSIC (1995) Heterogeneous responses of small mammals to an El Niño southern oscillation event in northcentral semiarid Chile and the importance of ecological scale. Journal of Mammalogy 76: 580-595.

MIKHAIL WZA (1993) Effect of soil structure on soil fauna in a desert wadi in southern Egypt. Journal of Arid Environments 24: 321-331.

MORENO R, J MORENO, JC ORTIZ, P VICTORIANO \& F TORRES-PÉREZ (2002) Herpetofauna del Parque Nacional Llanos de Challe (III Región, Chile). Gayana Zoología (Chile) 66: 7-10.

MORONI J (1972) Irrupción de Elasmoderus rabiosus (Lierbermann) en el Norte Chile (Acrididae, Chilacridinae). Noticiario Mensual del Museo Nacional Historia Natural (Chile) 192: 3-6.

MUÑOZ M, H NÚÑEZ \& J YÁÑEZ (1996) Libro rojo de los sitios prioritarios para la conservación de la diversidad biológica. Ministerio de Agricultura, Corporación Nacional Forestal, Santiago, Chile. 203 pp.

MUÑOZ-SCHICK M, R PINTO, A MESA \& A MOREIRAMUÑOZ (2001) "Oasis de neblina" en los cerros costeros del sur de Iquique, región de Tarapacá, Chile, durante el evento El Niño 1997-1998. Revista Chilena de Historia Natural 74: 389-405.

NEWELL IM \& L TEVIS (1960) Angelothrombium pandorae n.g., n. sp. (Acari, Trombidiidae), and notes on the biology of the giant red velvet mites. Annals of the Entomological Society of America 53: 293-304. 
PARMENTER RR, CA PARMENTER \& CD CHENEY (1989a) Factors influencing microhabitat partitioning among coexisting species of arid-land darkling beetles (Tenebrionidae): Behavioral response to vegetation architecture. Southwestern Naturalist 34: 319-329.

PARMENTER RR, CA PARMENTER \& CD CHENEY (1989b) Factors influencing microhabitat partitioning among coexisting species of arid-land darkling beetles (Tenebrionidae): temperature and water conservation. Journal of Arid Environments 17: 57-67.

PÉFAUR JE, JL YAÑEZ \& FM JAKSIC (1979) Biological and environmental aspects of a mouse outbreak in the semi-arid region of Chile. Mammalia 43: 313322.

PEÑA LE (1966) Catálogo de los Tenebrionidae (Coleoptera) de Chile. Entomologische Arbeiten aus dem Museum George Frey (Alemania) 17: $397-$ 453.

PEÑA LE (1980) Aporte al conocimiento de los tenebriónidos de América del Sur (Coleoptera: Tenebrionidae). Revista Chilena de Entomología 10: 37-59.

PIZARRO-ARAYA J \& GE FLORES (2004) Two new species of Gyriosomus Guérin-Méneville from Chilean coastal desert (Coleoptera: Tenebrionidae: Nycteliini). Journal of the New York Entomological Society 112: 121-126.

PIZARRO-ARAYA J \& V JEREZ (2004) Distribución geográfica del género Gyriosomus GuérinMéneville, 1834 (Coleoptera: Tenebrionidae): una aproximación biogeográfica. Revista Chilena de Historia Natural 77: 491-500.

POLIS GA, SD HURD, CT JACKSON \& F SÁNCHEZ PIÑERO (1997) El Niño effects on the dynamics and control of an island ecosystem in the Gulf of California. Ecology 78: 1884-1897.

ROGERS LE, NE WOODLEY, JK SHELDON \& PA BEEDLOW (1988) Diets of darkling beetles (Coleoptera: Tenebrionidae) within a shrub-steppe ecosystem. Annals of the Entomological Society of America 81: 782-791.

ROMERO H \& AM GARRIDO (1985) Influencias genéticas del fenómeno El Niño sobre los patrones climáticos de Chile. Investigación Pesquera (Chile) 32: 19-35

SANTIBÁÑEZ F \& J URIBE (1999) Origen, variabilidad y aspectos agroclimáticos de las sequías en Chile. En: Norero A \& C Bonilla (eds) Las sequías en Chile: causas, consecuencias y mitigación: 23-32. Ediciones Universidad Católica de Chile, Santiago, Chile.

SCHUMACHER A \& WG WHITFORD (1976) Spatial and temporal variation in Chihuahuan desert ant faunas. Southwestern Naturalist 21: 1-8.

SEELY MK (1979) Irregular fog as a water source for desert dune beetles. Oecologia 42: 213-227.

SEMIDA FM, MS ABDEL-DAYEM, SM ZALAT \& FS GILBERT (2001) Habitat heterogeneity and altitudinal gradients in relation to beetle diversity in south Sinai, Egypt. Egyptian Journal of Biology 3: 137-146.

SHELDON JK \& LE ROGERS (1984) Seasonal and habitat distribution of tenebrionid beetles in shrub- steppe communities of the Hanford Site in eastern Washington. Environmental Entomology 13: 214220

SHMIDA A, M EVENARI \& I NOY MEIR (1985) Hot deserts ecosystems: an integrated view. En: Evenari M, I Noy-Meir \& DW Goodall (eds) Hot deserts and arid shrublands: 379-387. Elsevier Science Publishers B.V., Amsterdam, The Netherlands.

SLOBODCHIKOFF CN (1983) Water balance and temperature preferences, and their role in regulating activity times of tenebrionid beetles. Oikos 40: 113119

SNELLING RR \& JH HUNT (1975) The ants of Chile (Hymenoptera: Formicidae). Revista Chilena de Entomología 9: 63-129.

STAPP P (1997) Microhabitat use and community structure of darkling beetles (Coleoptera: Tenebrionidae) in shortgrass prairie: effects of season, shrub cover and soil type. American Midland Naturalist 137: 298-311.

THOMAS DB (1979) Patterns in the abundance of some tenebrionid beetles in the Mojave desert. Environmental Entomology 8: 568-574.

TOLEDO X \& E ZAPATER (1989) Geografía general y regional de Chile. Editorial Universitaria, Santiago, Chile. $251 \mathrm{pp}$.

TORRES-CONTRERAS HT (2001) Antecedentes biológicos de hormigas presentes en Chile publicados en revistas científicas nacionales y extranjeras durante el siglo XX. Revista Chilena de Historia Natural 74: 653-668.

UNDERWOOD AJ (1999) Experiments in ecology: their logical design and interpretation using analysis of variance. Cambridge University Press, Cambridge, United Kingdom. vii + 504 pp.

VIDIELLA PE, JJ ARMESTO \& JR GUTIÉRREZ (1999) Vegetation changes and sequential flowering after rain in the southern Atacama desert. Journal of Arid Environments 43: 449-458.

WALLWORK JA (1982) Desert soil fauna. Praeger Scientific, London, United Kingdom. x + 296 pp.

WHITFORD WG (1978) Structure and seasonal activity of Chihuahuan desert ant communities. Insectes Sociaux 25: 79-88

WHITFORD WG (1999) Seasonal and diurnal activity patterns in ant communities in a vegetation transition region of southeastern New Mexico (Hymenoptera: Formicidae). Sociobiology 34: 477 491.

WHITFORD WG, E DEPREE \& P JOHNSON (1980) Foraging ecology of two Chihuahuan desert ant species: Novomessor cockerelli and Novomessor albisetosus. Insectes Sociaux 27: 148-156.

WISE DA (1985) Negative correlation between numbers of a darkling beetle and a carabid predator. Southwestern Naturalist 30: 148-150.

WORK TT, CM BUDDLE, LM KORINUS \& JR SPENCE (2002) Pitfall trap size and capture of three taxa of litter-dwelling arthropods: implications for biodiversity studies. Environmental Entomology 31: 438-448.

YUNGER JA, PL MESERVE, JR GUTIÉRREZ (2002) Small mammal foraging behavior: mechanisms for coexistence and implication for population dynamics. Ecological Monographs 72: 561-577.

Editor Asociado: Audrey Grez

Recibido el 10 de diciembre de 2004; aceptado el 6 de junio de 2005 